\title{
Simultaneous observation of the magnetic and electric behavior in a correlated system near a metal-semiconductor transition: ESR in pellets of conducting polymers
}

\author{
J. M. Kondo and L. Walmsley \\ Departamento de Física, Instituto de Geociências e Ciências Exatas, Universidade Estadual Paulista, Caixa Postal 178, CEP 13500-970 \\ Rio Claro, SP, Brazil \\ C. Rettori and M. S. Sercheli \\ Instituto de Física “Gleb Wataghin,” UNICAMP, CEP 13083-970 Campinas, SP, Brazil
}

A. A. Correa and E. C. Pereira

Departamento de Química, Universidade Federal de São Carlos, Caixa Postal 676, CEP 13560-970 São Carlos, SP, Brazil

(Received 10 February 2009; revised manuscript received 8 May 2009; published 8 July 2009)

\begin{abstract}
Electron spin resonance (ESR) experiments show, at the metal-semiconductor transition temperature of a conducting polymer, the distinct contributions of the disordered and crystalline regions. In the more disordered regions of the polymer the polarons experience an antiferromagnetic coupling. As the level of disorder decreases, when small crystalline regions appear, there is a tendency, in some temperature range, for the polarons to interact ferromagnetically. For more ordered regions or crystalline regions of larger sizes, there is a competition between localized ferromagnetic coupled polarons and delocalized ones, that is, between localization and delocalization. The possibility to fit the ESR data for one of the samples using two Dysonian lines, one for each phase, allows one to follow the general behavior of the microwave conductivity as a function of the temperature. The semiconducting behavior of the disordered phase is clearly observed, as well as the true metallic behavior of the crystalline phase revealed by the increase in conductivity with the decrease in temperature.
\end{abstract}

DOI: 10.1103/PhysRevB.80.014410 PACS number(s): 76.30.Pk, 82.35.Cd, 71.70.Gm, 71.30. $+\mathrm{h}$

\section{INTRODUCTION}

The approaching model where a conjugated polymer may be considered as a correlated insulator has been emphasized by Kivelson and Emery ${ }^{1,2}$ assuming that conjugated polymers would be good metals if the Peierls instability would not dimerize them. Thus, the doping that transforms a conjugated polymer in a conducting polymer is generally seen as a topological doping. In a recent $\operatorname{article}^{3}$ we have discussed the connection between the formation of defects, polarons and bipolarons, in conjugated polymers with a nondegenerate ground state, with the observed magnetic susceptibility behavior. We have concluded that our magnetization and electron spin resonance (ESR) data in conducting polymer pellets could be explained assuming ferromagnetic (FM) and antiferromagnetic (AFM) interacting spin polarons. The observation of AFM correlated polarons in doped plasticized polyaniline films ${ }^{4}$ allowed us to propose a more general scenario for the polaronic interaction in conducting polymers. We have suggested that small crystalline portions with FM coupling are surrounded by disordered polymers with AFM interaction. We argued that this behavior is due to an energy balance requirement. The energy of the crystalline arrangement is lower than that of the disordered polymer, requiring the magnetic moment pairing to be FM in order to increase the energy and match the energy of the surrounded disordered polymer. In the case of highly doped samples, the balance of energy in the ordered regions is provided by the kinetic energy. The first measurements in doped polyacetylene have pointed out the need to consider the heterogeneous character of conduction in conducting polymers. Park et al. ${ }^{5}$ suggested that the metallic state could be described by metallic strands separated by thin potential barriers. In order to understand thermoelectric power data of highly conductivity samples Kaiser ${ }^{6}$ proposed a model of metallic fibrils separated by electrical barriers after being extended to treat smaller metallic regions. ${ }^{7}$ The model of metallic islands, connected via low conducting regions, has also been previously proposed ${ }^{8}$ based on magnetic susceptibility measurements. This "metallic island" model has shown to be appropriated to explain the charge transport properties in partially crystalline polyanilines, suggesting that doped polyaniline films are composed of ordered crystalline regions, described by three-dimensional metallic states, connected through amorphous regions of polymer chains. ${ }^{9,10}$ This heterogeneous conductivity model has also been inferred from NMR measurements in polyaniline by Beau et al. ${ }^{11}$ However, from the measurement of intrachain and interchain diffusion rates, they concluded for a strongly anisotropic quasi-onedimensional (quasi-1D) motion for the spin carriers. Moreover, in heavily doped polyaniline films, Kahol et al. ${ }^{12}$ using ESR showed the influence of moisture in the appearance of an intermediate region connecting the metallic islands to the amorphous regions.

In this paper we shall show how X-band ESR measurements in pressed pellets of doped poly(3-methylthiophene), performed in the temperatures range between 120 and $300 \mathrm{~K}$, will allow us to obtain, simultaneously, information on the correlated polarons and charge transport properties of the disordered and crystalline regions of the polymer. These experiments have the advantage of exploring different portions of the sample using a contactless technique. Basically, we 
show that all the previous assumptions about the coupling of the polarons in the different parts of the polymers are confirmed and further enriched by the information on charge transport. For this study we used two different samples: sample 1, synthesized at room temperature where the distinction between the phases is less evident, and sample 2, synthesized at $5{ }^{\circ} \mathrm{C}$, where a clear difference in the behavior of the ESR parameters was observed for both phases. Changes in conductivity of poly(3-methylthiophene) due to similar variations in the temperature of synthesis have been observed by Sato et al. ${ }^{13}$ The increase in molecular weight and crystallinity and the decrease in the number of defects in polythiophene derivatives synthesized at low temperatures have also been reported by $\mathrm{Hu}$ and $\mathrm{Xu}^{14}$ Similar findings concerning increase in molecular weight and also in crystallinity have been reported for polyaniline. ${ }^{15,16}$ It is interesting to note that while $\mathrm{x}$-ray diffraction does not allow a better structural characterization of disordered samples, ESR provides indirect structural information. Besides, for the thin sample 1 the data could be fitted with two Lorentzian lines, while for the thick sample 2, two Dysonian lines were used, ${ }^{17}$ providing the additional information on charge transport. The semiconducting behavior of the disordered phase is clearly observed and the true metallic behavior of the crystalline phase as well, which is revealed by the increase in conductivity with the decrease in temperature. The separate behavior of these two phases is directly shown and not indirectly inferred from heterogeneous conductivity models.

\section{EXPERIMENTAL DETAILS}

Poly(3-methylthiophene) was electrochemically synthesized in acetonitrile containing $0.1 M$ of $\mathrm{LiClO}_{3}$ and the same amount of the monomer. The pellets were uniaxially pressed at $6.2 \mathrm{kbar}$. Two samples, named sample 1 and sample 2, were synthesized. Sample 1 of $60 \mu \mathrm{m}$ thick was synthesized at $20{ }^{\circ} \mathrm{C}$ and sample 2 of $210 \mu \mathrm{m}$ thick was synthesized at $5{ }^{\circ} \mathrm{C}$. With a much lower temperature of synthesis sample 2 is less disordered and has a doping level around $12 \%$ determined by electron dispersive spectroscopy measurements from the $\mathrm{Cl} / \mathrm{S}$ ratio. The doping level of sample 1 is around $8 \%$. X-ray diffraction experiments on samples prepared in conditions similar to samples 1 and 2 did not show any interesting feature except for the amorphous spectrum that indicates a low crystalline portion in both cases. X-band ESR experiments were performed in a Bruker ELEXIS spectrometer with the samples encapsulated in a special quartz ESR tube in helium atmosphere.

\section{RESULTS AND DISCUSSION}

The ESR spectra of sample 1 were best fitted with a combination of two Lorentzian lines. Lorentzian lines have also been found in doped poly(3-methylthiophene) films for similar doping levels. ${ }^{18,19}$ For sample 2 the best fittings were for a combination of two Dysonian lines. In fact, the Dysonian lines were written as a combination of absorption and dispersion of Lorentzian lines. This procedure is very accurate to describe Dysonian lines in the limit of thin samples. ${ }^{20}$ In Fig.

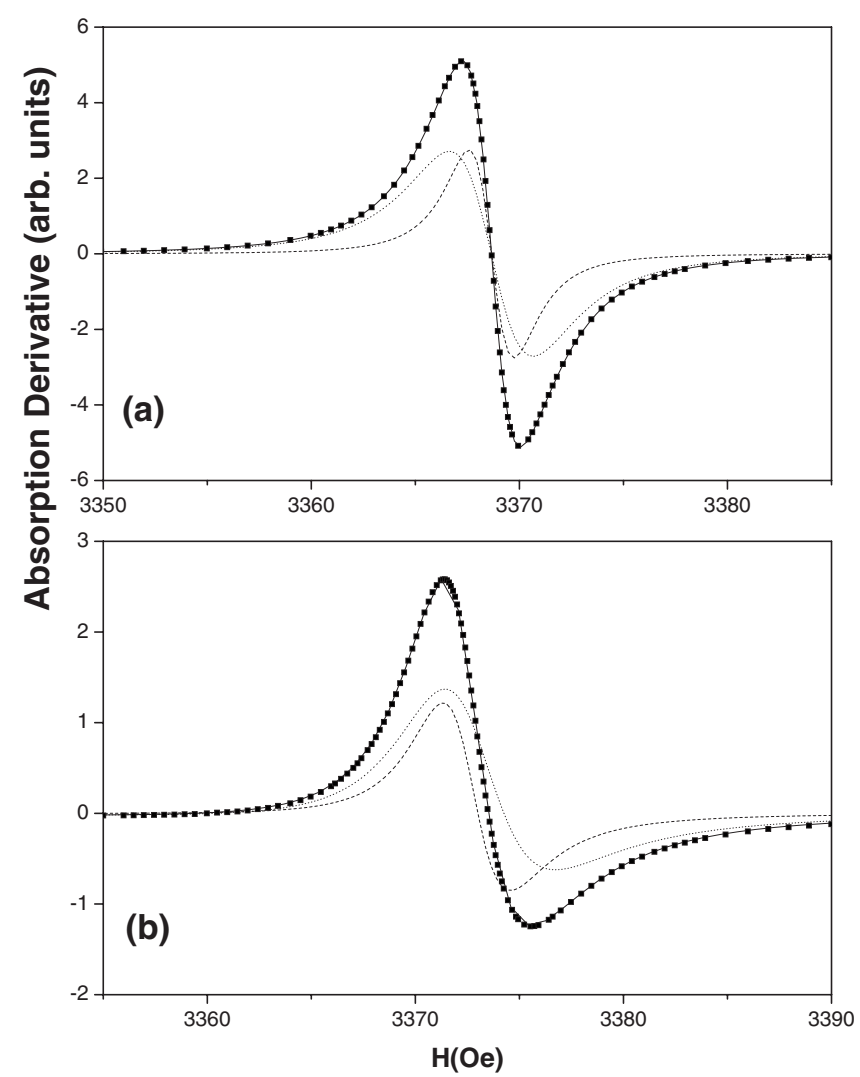

FIG. 1. Room temperature X-band ESR spectra: (a) for sample 1 (squares) fitted to a combination of two Lorentzian lines (full line) showing each Lorentzian line (dashed line and dotted line); (b) for sample 2 (circles) fitted to a combination of two Dysonian lines (full line) showing each Dysonian line (dashed line and dotted line).

1 we show the room-temperature ESR spectra for both samples. For sample 1 in Fig. 1(a), we show the ESR spectrum (full squares) fitted (full line) to a combination of two Lorentzian lines (dashed-dotted-dashed lines). For sample 2 in Fig. 1(b) everything is similar except that the Lorentzian lines are replaced by two Dysonian lines. Fitting a magnetic resonance spectrum with more than one component always implies dissimilar magnetic moments at least from the magnetic point of view. This multicomponent fitting implies that different sets of magnetic moments are seeing different local magnetic fields that could be due to dipolar, exchange, or nuclear (dipolar or quadrupolar) magnetic moment interaction. Because all these interactions are dependent not only on the distance between the magnetic moments but also on the way they are arranged, we can establish a connection between different magnetic environments and the organization of the surroundings of a magnetic moment. From the fitting of sample 1 with the two Lorentzian lines the $g$ values and linewidths could be obtained. They are shown in Fig. 2. In Fig. 2(a) the $g$ values for both Lorentzian lines are shown. They are about the same in the whole temperature interval. In Fig. 2(b) the linewidths for both lines are shown. For the broader line (full squares) an increase in the linewidth with temperature starting around $275 \mathrm{~K}$ can be observed. For the narrower line this behavior starts around $250 \mathrm{~K}$. This behavior is expected for the Elliott's mechanism ${ }^{21}$ of spin-orbit 


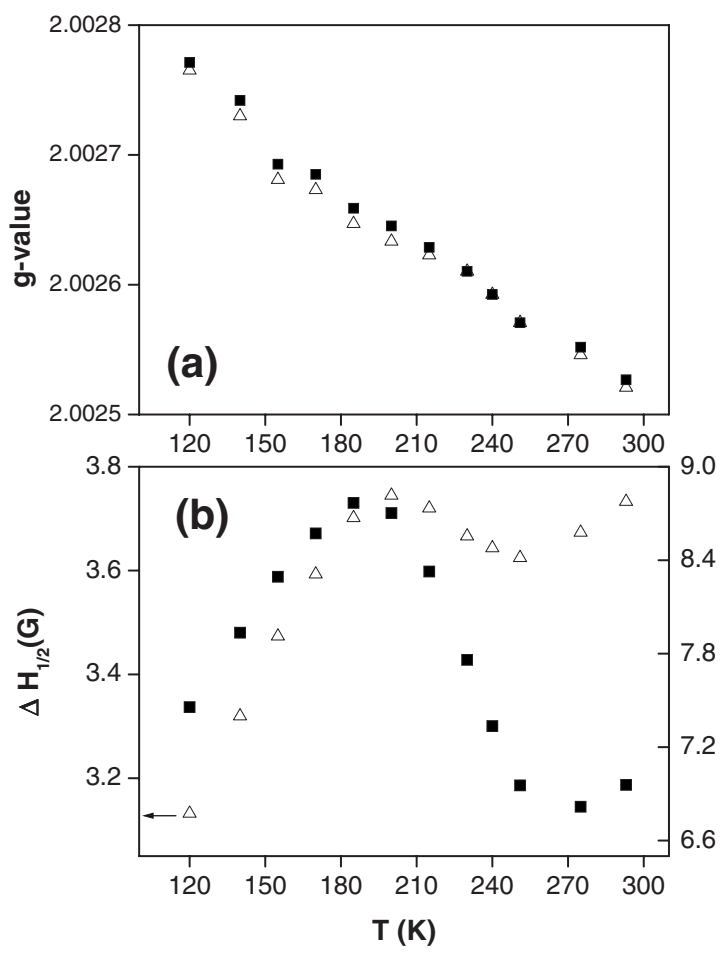

FIG. 2. (a) Gyromagnetic factors versus temperature for the broad Lorentzian line (full squares) and the narrow Lorentzian line (open triangles) for the spectra of sample 1. (b) Linewidths for the same lines.

relaxation in a conducting system. According to this mechanism there is a relation of proportionality between the relaxation time $\tau_{S}$ associated with the spin-flip scattering and the relaxation time $\tau_{R}$ characteristic of the electrical resistivity. Above the Debye temperature this relation can be written as $\Delta H \alpha \rho$, where $\rho$ is the resistivity. On the metallic side of the metal-semiconductor transition and at high temperatures the resistivity increases with temperature. In the semiconductor part of the transition, the conduction is via hopping and there is a decrease in the linewidth with the increase in temperature, evidencing the decrease in resistivity with the increase in temperature. A linear increase behavior has been observed by Schärli et al. ${ }^{19}$ for highly doped poly(3-methylthiophene) films. They found that the higher the doping level, the lower the temperature at which the increase in the linewidth with temperature starts. Similarly, we can assume that the difference in temperatures below which the increase in linewidth with increasing temperature starts to be observed indicates that the broad line corresponds to a slightly more disordered portion of the sample with the metal-semiconductor transition taking place at higher temperatures for this part of the system. The observed semiconductor behavior, via the Elliott's mechanism of spin-orbit relaxation, can be observed up to around $210 \mathrm{~K}$ for the narrow line and slightly below this temperature for the broad line. At lower temperatures, the decrease in the linewidth with temperature could be due to exchange narrowing effects. Although for low dimensional systems the expected line shape due to exchange effects is not Lorentzian, the Lorentzian line shape is recovered by considering the effect of a weak interchain coupling. ${ }^{22}$ In

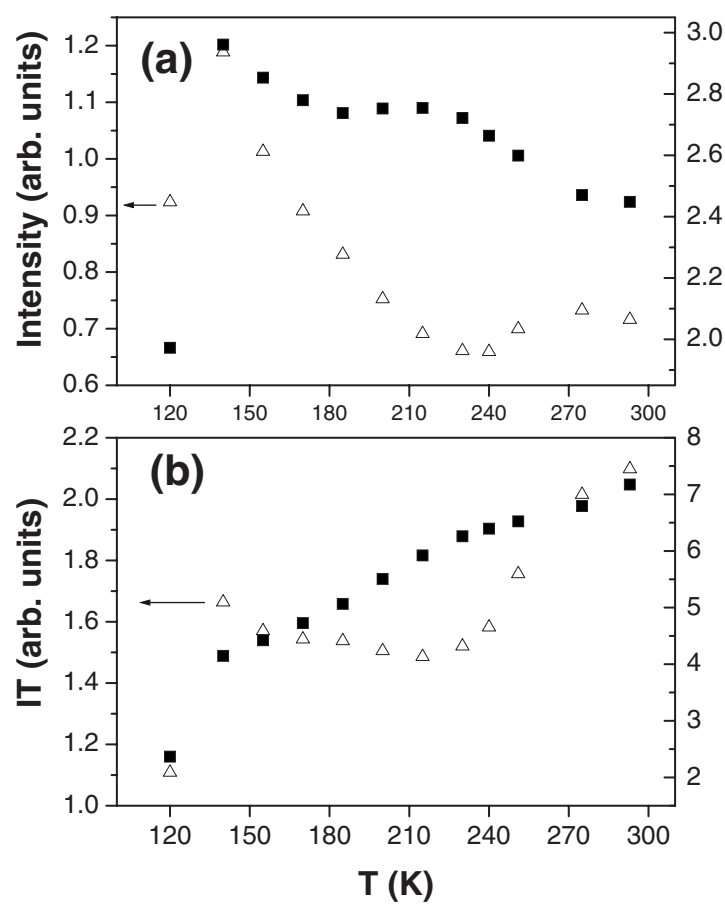

FIG. 3. (a) Intensity versus temperature for the broad Lorentzian line (full squares) and the narrow Lorentzian line (open triangles) for the spectra of sample 1 . (b) Intensity times temperature $(I \times T)$ versus $T$ for both lines.

Fig. 3(a) the ESR intensity is shown for both lines. The temperature independent Pauli behavior is observed in the metallic phase, which corresponds to the small region of temperatures for which the linewidth shows the typical behavior of Elliott's mechanism for a metallic system. Below those temperatures the intensities do not increase monotonically. Instead, maxima and minima are seen for the narrow line. In order to have a better understanding of this behavior, in Fig. 3 (b) the product of the intensity, $I$, times the temperature, $T$, $(I \times T)$ is shown as a function of the temperature. From these data some interesting conclusions can be obtained. First, a typical Curie-law behavior is not seen for any of the lines. For a Curie-law behavior to be observed, a temperature independent line should be seen in the $(I \times T)$ versus $T$ plot. Due to this fact, we suggest that for both lines, the magnetic moments are somehow correlated. For the broad and more intense line (full squares), corresponding to the slightly more disordered portion of the sample in the semiconducting phase, the magnetic moments are AFM correlated ${ }^{23}$ at all temperatures. For the narrow line (open triangles) we observe that, between 210 and $130 \mathrm{~K}$, there is a tendency to a FM coupling.

In order to show that this tendency to FM coupling is real, we shall discuss the ESR data for sample 2. In the case of sample 2, as it has been shown in Fig. 1(b), all the spectra were fitted with two Dysonian lines. The general expression of a Dysonian ${ }^{17}$ line shape depends on two parameters, $\lambda$ $=d / \delta(d$ is the thickness of the sample and $\delta$ is the skin depth of the microwave field) and $R=\left(T_{D} / T_{2}\right)^{1 / 2}\left(T_{D}\right.$ is the diffusion time of the spin across the skin depth and $T_{2} \approx T_{1}$ is the spin relaxation time). For our experimental conditions we 


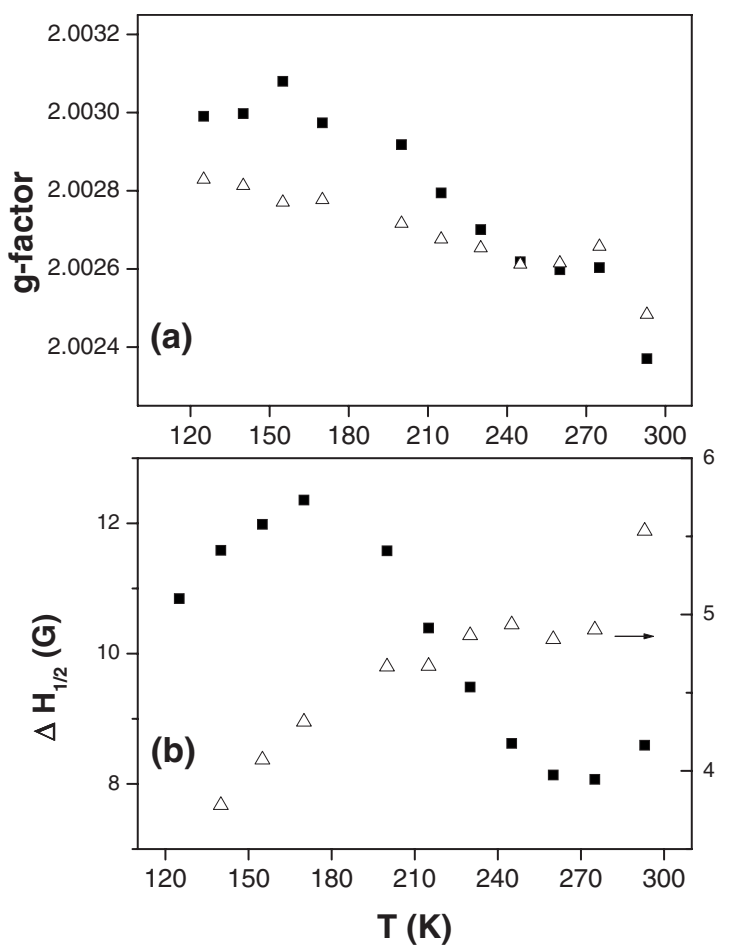

FIG. 4. (a) Gyromagnetic factors versus temperature for the broad Dysonian line (full squares) and the narrow Dysonian line (open triangles) for the spectra of sample 2. (b) Linewidths for the same lines.

have $d \leq \delta$, thus, we are in the limit of thin sample of Dyson's theory. In this limit the line shape does not depend on $R$, it is only a function of $\lambda$. The Lorentzian lines obtained for sample 1 are just the extreme case of this thin sample limit. For sample 2 the asymmetrical lines are expected due to the increase in the doping level and thickness. Besides, in this limit the Dysonian line shape can be very well described by a combination of absorption and dispersion of Lorentzian lines..$^{20}$ This is the procedure we adopted to fit the line shapes of sample 2. In Figs. 4(a) and 4(b) the $g$ values and linewidths, respectively, are shown for the broad Dysonian line (full squares) and narrow Dysonian line (open triangles). Unlike sample 1, in sample 2 the $g$ values of the broad and narrow Dysonian lines take different values below $240 \mathrm{~K}$. This fact evidences that a clear phase separation can now be observed in sample 2, with distinct magnetic environments for the magnetic moments. If we compare the temperature dependence of the linewidths for sample 2 with that of sample 1, we find that only the broad line of sample 2 follows the metal-semiconductor transition around $270 \mathrm{~K} \mathrm{ob}-$ served in sample 1, which was attributed to Elliott's mechanism. For the narrow Dysonian line the typical behavior of the semiconducting phase is not clearly observed. In Fig. 5 (a) the intensity versus temperature is shown for sample 2 and the intensity times temperature $(I \times T)$ versus $T$ is shown in Fig. 5(b).

In Fig. 5(a) a transition can be observed for the narrow and less intense line (open triangles) around $280 \mathrm{~K}$. For this line a Pauli behavior can be seen between 250 and $210 \mathrm{~K}$. This behavior is in agreement with the observation of a lin-

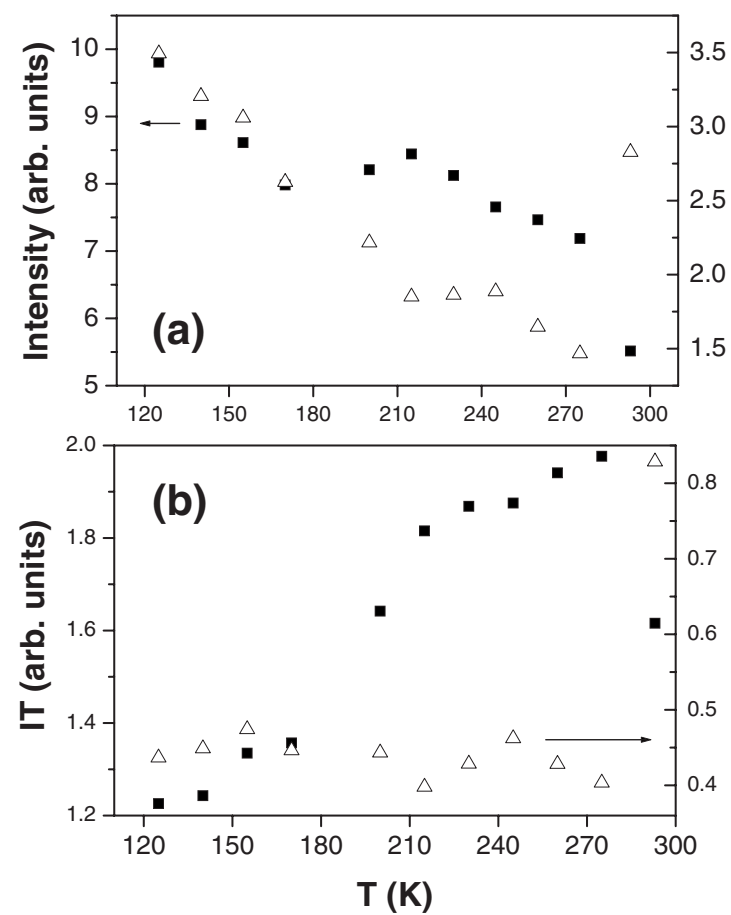

FIG. 5. (a) Intensity versus temperature for the broad Dysonian line (full squares) and the narrow Dysonian line (open triangles) for the spectra of sample 2. (b) Intensity times temperature $(I \times T)$ versus $T$ for both lines.

ear increase in the linewidth in this range of temperature, characteristic of Elliott's relaxation mechanism for metallic systems. In Fig. 5(b) the $(I \times T)$ versus $T$ data show again evidences for FM correlated magnetic moments up to around $150 \mathrm{~K}$. For the broad line a behavior similar to that of the more disordered phase of sample 1 is observed. Consequently, this line probably corresponds to the more disordered phase of sample 2. Due to the fact that the lines are Dysonians, one more ESR parameter can be used to give information about the system. This parameter is the asymmetry ratio $(A / B)$ of the absorption derivative line. The experimental conditions allow us to assume that we are in the thin sample limit of Dyson's theory. In such a situation the sample thickness $(d)$ is small or comparable to the skin depth of the microwave field $(\delta)$. But, due to the fact that we have two different phases in the sample, we are not able to obtain the conductivity at the microwave frequency according to the procedure and the relation discussed in Ref. 20 (MKS units),

$$
\lambda=\frac{d}{\delta}=\left(\frac{d^{2}}{\pi \nu_{0} \mu \sigma}\right)^{1 / 2} .
$$

This is because we cannot attribute a corresponding thickness to each phase of the sample. In Eq. (1) $\nu_{0}$ is the microwave frequency, $\mu$ is the magnetic permeability, and $\sigma$ is the microwave conductivity. In spite of this, the general behavior of the microwave conductivity can be followed as a function of temperature. By this we mean to follow the temperature dependence of the $(A / B)$ ratio for each Dysonian line. It is important to note that a large $(A / B)$ ratio for one of the phases does not mean that the microwave conductivity is 


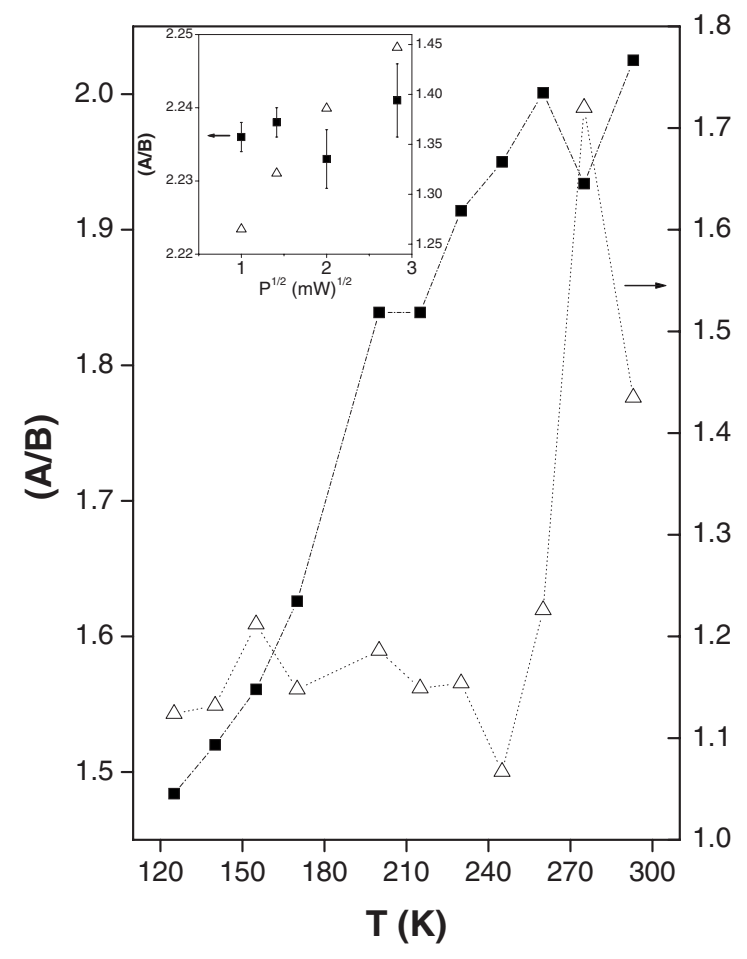

FIG. 6. Absorption derivative asymmetry ratio $(A / B)$ versus temperature for the broad (full squares) and the narrow (open triangles) Dysonian lines of sample 2. Inset: room temperature values of $(A / B)$ versus square root of the microwave power for both lines.

larger for that phase. It would be necessary to have the same thickness for both phases to be able to compare the $(A / B)$ ratios. In Fig. 6 the $(A / B)$ ratio is shown as a function of the temperature for the two phases. For the more disordered phase (full squares) the metal-semiconductor transition around $280 \mathrm{~K}$ can be visualized, and below this temperature, the semiconducting behavior is observed. For the more ordered phase (open triangles), which we attribute to the crystalline portions of the polymer, successive transitions are observed. A metallic behavior can be seen between 250 and 210 $\mathrm{K}$. This is in agreement with the Pauli behavior observed in Fig. 5 for the same temperature interval. In spite of the small number and dispersion of the data points, one may notice that in the crystalline regions the temperature behavior resembles the 1D conductivity ${ }^{24}$ in agreement with Ref. 11 . We can also observe that for sample 2 the decrease in the $A / B$ values when the temperature decreases suggests that the Lorentzian line limit $(A / B=1)$ is close to be reached. The conclusion about the more 1D-like character of the conductivity of the more ordered phase is reinforced by the roomtemperature measurements of the microwave power dependence of the line asymmetry $(A / B)$ in the power range where line saturation effects are not significant. For the microwave to penetrate into the sample, both the microwave electric and magnetic fields of the electromagnetic wave must enter into the sample. In our experimental condition the sample is placed into a $\mathrm{TE}_{102}$ microwave cavity where the microwave electric field component is much lower than the magnetic component but it is not negligible. The average value of the square of the magnetic microwave field for a rectangular $\mathrm{TE}_{102}$ microwave cavity is given by ${ }^{25}$

$$
\left\langle H_{1}^{2}\right\rangle \approx 0.8 \times 10^{-3} Q_{L} P_{u} .
$$

$Q_{L}$ is the loaded quality factor and $P_{u}$ is the unloaded microwave power. The power absorbed by the sample is also proportional to the mean value of the square of the microwave electric field. ${ }^{26}$ In this way, a plot of the line asymmetry $(A / B)$ versus square root of the microwave power gives the overall behavior of the microwave conductivity as a function of the microwave electric field. In the inset of Fig. 6 we show these data for both phases of sample 2, disordered region (full squares) and crystalline portion (open triangles). For the more disordered phase, within the error bar, an Ohmic behavior is observed. However, for the crystalline portions, a clear nonlinear behavior can be seen. Since these results are obtained from a contactless measurement, this nonlinear behavior reinforces the quasi-1D character of the charge transport already observed in systems with low dimensionality. ${ }^{27}$

\section{CONCLUSIONS}

In this paper we have shown how ESR X-band measurements in a restricted range of temperatures allowed us to obtain simultaneously magnetic and electric information on both disordered and crystalline phases of doped pellets of poly(3-methylthiophene). The magnetic information can be summarized as follows: in the more disordered regions of the polymer the polarons experience an AFM coupling. As the level of disorder decreases when small crystalline regions appear, there is a tendency, in some temperature range, for the polarons to interact FM. For more ordered regions or crystalline regions of larger sizes, there is a competition between localized FM coupled polarons and delocalized ones, that is, between localization and delocalization. These conclusion have been previously obtained based on magnetization and ESR data in partially reduced poly(3methythiophene) pellets ${ }^{3}$ and doped plasticized polyaniline films. ${ }^{4}$ The interesting thing about our data is that the fitting of the ESR data for sample 2 with two Dysonian lines enables us to follow the behavior of the microwave conductivity as a function of temperature in each phase of the sample. This separation is obtained in a contactless measurement. The semiconducting behavior of the disordered phase is clearly observed, as well as the true metallic behavior of the crystalline phase revealed by the increase in conductivity with the decrease in temperature. We argued that the conductivity in these metallic islands seems to be more one dimension in character. This conclusion is reinforced for the evidences of nonlinear behavior of the microwave conductivity extracted from the ESR contactless measurements as a function of the microwave power.

\section{ACKNOWLEDGMENTS}

This work was partially supported by Fundação de Amparo à Pesquisa do Estado de São Paulo (FAPESP) and Conselho Nacional de Desenvolvimento Científico e Tecnológico (CNPq). 
${ }^{1}$ S. A. Kivelson and A. J. Emery, Synth. Met. 65, 249 (1994).

${ }^{2}$ S. A. Kivelson and A. J. Emery, Synth. Met. 80, 151 (1996).

${ }^{3}$ O. R. Nascimento, A. J. A. de Oliveira, E. C. Pereira, A. A. Correa, and L. Walmsley, J. Phys.: Condens. Matter 20, 035214 (2008).

${ }^{4}$ D. Djurado, A. Pron, J.-P. Travers, J. G. S. Duque, P. G. Pagliuso, C. Rettori, D. L. Chinaglia, and L. Walmsley, J. Phys.: Condens. Matter 20, 285228 (2008).

${ }^{5}$ Y.-W. Park, A. J. Heeger, M. A. Druy, and A. G. MacDiarmid, J. Chem. Phys. 73, 946 (1980).

${ }^{6}$ A. B. Kaiser, Phys. Rev. B 40, 2806 (1989).

${ }^{7}$ A. B. Kaiser, G. U. Flanagan, D. M. Stewart, and D. Beaglehole, Synth. Met. 117, 67 (2001).

${ }^{8}$ J. M. Ginder, A. F. Richter, A. G. MacDiarmid, and A. J. Epstein, Solid State Commun. 63, 97 (1987).

${ }^{9}$ Z. H. Wang, E. M. Scherr, A. G. MacDiarmid, and A. J. Epstein, Phys. Rev. B 45, 4190 (1992).

${ }^{10}$ J. Joo, S. M. Long, J. P. Pouget, E. J. Oh, A. G. MacDiarmid, and A. J. Epstein, Phys. Rev. B 57, 9567 (1998).

${ }^{11}$ B. Beau, J. P. Travers, and E. Banka, Synth. Met. 101, 772 (1999).

${ }^{12}$ P. K. Kahol, A. J. Dyakonov, and B. J. MacCormick, Synth. Met. 89, 17 (1997).
${ }^{13}$ M. Sato, T. Susumo, and K. Kaeriyama, Synth. Met. 14, 279 (1986).

${ }^{14}$ X. Hu and L. Xu, Polymer 41, 9147 (2000).

${ }^{15}$ L. H. C. Mattoso, A. G. Macdiarmid, and A. J. Epstein, Synth. Met. 68, 1 (1994).

${ }^{16}$ J. Stejskal, A. Riede, D. Hlavata, J. Prokes, M. Helmstedt, and P. Holler, Synth. Met. 96, 55 (1998).

${ }^{17}$ F. J. Dyson, Phys. Rev. 98, 349 (1955).

${ }^{18}$ Y. Harima, T. Eguchi, K. Yamashita, K. Kojima, and M. Shiotani, Synth. Met. 105, 121 (1999).

${ }^{19}$ M. Schärli, H. Kiess, G. Harbeke, W. Berlinger, K. W. Blazey, and K. A. Müller, Synth. Met. 22, 317 (1988).

${ }^{20}$ L. Walmsley, J. Magn. Reson., Ser. A 122, 209 (1996).

${ }^{21}$ R. J. Elliott, Phys. Rev. 96, 266 (1954).

${ }^{22}$ A. Bencini and D. Gateschi, Electron Paramagnetic Resonance of Exchange Coupled Systems (Springer Verlag, Berlin, 1990).

${ }^{23}$ A. Abragam and B. Bleaney, Electron Paramagnetic Resonance of Transitions Ions (Dover, New York, 1986), p. 507.

${ }^{24}$ A. B. Kaiser, Rep. Prog. Phys. 64, 1 (2001).

${ }^{25}$ C. P. Poole, Jr., Electron Spin Resonance, 2nd ed. (Dover, New York, 1996).

${ }^{26}$ L. Walmsley, Phys. Rev. B 46, 6256 (1992).

${ }^{27}$ G. Grüner, Rev. Mod. Phys. 60, 1129 (1988). 